\title{
VEHICULAR MOTION EXPERIMENT AND DATA RETRIEVAL OF A COMPACT FLOATING LIDAR SYSTEM
}

\author{
Qichao Wang ${ }^{1}$, Shengguang Qin ${ }^{3}$, Jiaping Yin ${ }^{3}$, Hongwei Zhang ${ }^{1}$, Tong Cui ${ }^{1}$, Songhua Wu ${ }^{1,2}$ \\ ${ }^{I}$ Ocean Remote Sensing Institute, College of Information Science and Engineering, Ocean University of \\ China, Qingdao 266100, China \\ ${ }^{2}$ Laboratory for Regional Oceanography and Numerical Modeling, Pilot National Laboratory for Marine \\ Science and Technology (Qingdao), Qingdao 266237, China \\ ${ }^{3}$ Leice Transient Technology Co. LTD, Qingdao 266100, China
}

*Email:wqc@stu.ouc.edu.cn

\begin{abstract}
Accurate and rapid observation of sea surface wind is important for the research of ocean dynamic prediction model, offshore wind resource assessment, air-sea interaction and flux. A compact floating coherent Doppler lidar system named WindMast 350-M was developed by Ocean University of China (OUC) and Leice Transient Technology Co. LTD (LEICE) for the observations of sea surface wind profiles. As an observation device installed on buoy platforms, the first vehicular motion experiment was conducted at Laoshan campus of Ocean University of China $\left(120.49^{\circ} \mathrm{E}, 36.16^{\circ} \mathrm{N}\right)$ on 06 and 12 March, 2019. During the first experiment, the wind profiles measured by the WindMast 350$\mathrm{M}$ were compared with the results from a well calibrated Ground-based Coherent Doppler lidar WindMast WP350. In this contribution, the systematic design and the specifications of 350-M are presented in detail. The preliminary results of the vehicular motion experiment are discussed as well.
\end{abstract}

Keywords: Floating Coherent Doppler lidar, Motion Correction, Data Retrieval and Validation.

\section{INTRODUCTION}

As an effective method for wind detection, Coherent Doppler lidar has the advantages of high accuracy, high spatial and temporal resolution. It is capable of low-altitude wind measurement. By combining buoy and wind lidar, floating lidar systems offer a great potential to offshore wind resources assessment, ocean dynamic prediction model research, air-sea interaction and flux study [1]. Compared with traditional fixed offshore meteorological masts, floating lidar system is cheaper and flexible. It also helps to improve the ability of sea surface wind detection under bad conditions, which compensates for the shortcomings of shipborne observation equipments.

Therefore, a compact floating lidar system WindMast 350-M was developed by the Ocean University of China (OUC) and Leice Transient Technology Co. LTD (LEICE). In this paper, the system design, the details and preliminary results of the first experiment are presented.

\section{METHODOLOGY}

\subsection{System design}

WindMast 350-M system consists of laser transmitter, scanning servo, optical receiver, signal collector and processor. All fiber and modular design are adopted to achieve the antivibration ability, thus improves the stability and reliability of whole system. To obtain the attitude and velocity information, a compact GPS/MEMS (Micro Electro Mechanical System) was equipped inside the lidar system.

Table 1. The specifications of the WindMast 350-M

\begin{tabular}{|c|c|}
\hline Qualification & Specification \\
\hline Wavelength & $1.5 \mu \mathrm{m}$ \\
\hline Pulse Energy & $12 \mu \mathrm{J}$ \\
\hline Pulse Duration & $100 / 200 \mathrm{~ns}$ \\
\hline $\begin{array}{c}\text { Pulse Repetition } \\
\text { Frequency }\end{array}$ & $10 \mathrm{kHz}$ \\
\hline Pitch Angle & $60^{\circ}$ \\
\hline $\begin{array}{c}\text { Radial Spatial } \\
\text { Resolution }\end{array}$ & $10 \mathrm{~m}$ \\
\hline Measurement Range & 40 to $350 \mathrm{~m}$ \\
\hline $\begin{array}{c}\text { Wind Profile Update } \\
\text { Rate }\end{array}$ & $1 \mathrm{~min}$ \\
\hline $\begin{array}{c}\text { Velocity Measurement } \\
\text { Range }\end{array}$ & $\pm 60 \mathrm{~m} / \mathrm{s}$ \\
\hline Accuracy of Velocity & $0.3 \mathrm{~m} / \mathrm{s}$ \\
\hline
\end{tabular}




\begin{tabular}{|c|c|}
\hline Power Consumption & $<80 \mathrm{w}$ \\
\hline Weight & $<30 \mathrm{~kg}$ \\
\hline Size & $452 * 351 * 430 \mathrm{~mm}$ \\
\hline Eye Safety Standards & EN60825-1:2007 \\
\hline
\end{tabular}

\subsection{Experiments design}

During the first vehicular motion experiment, an undulating area was selected at the Laoshan campus of OUC (Figure 1) in order to simulate/demonstrate the undulate motion of buoy. The WindMast 350-M system was carried by a pickup truck (Figure 2), moving on a circle route in clockwise direction constantly. For purpose of intercomparison, a ground-based Coherent Doppler lidar of the type WindMast WP350 was deployed nearby simultaneously (green star in Figure 1).

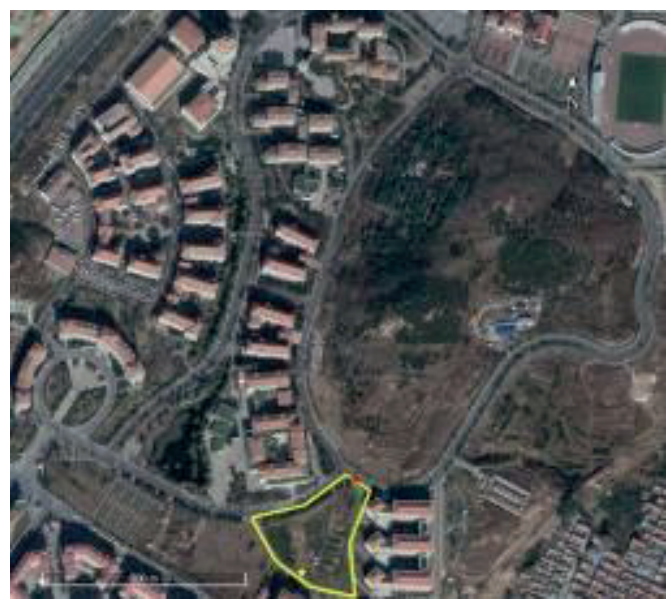

Figure 1. The Route of First Experiment (Yellow Line) and the Placement Location of WindMast WP350 (Green Star)

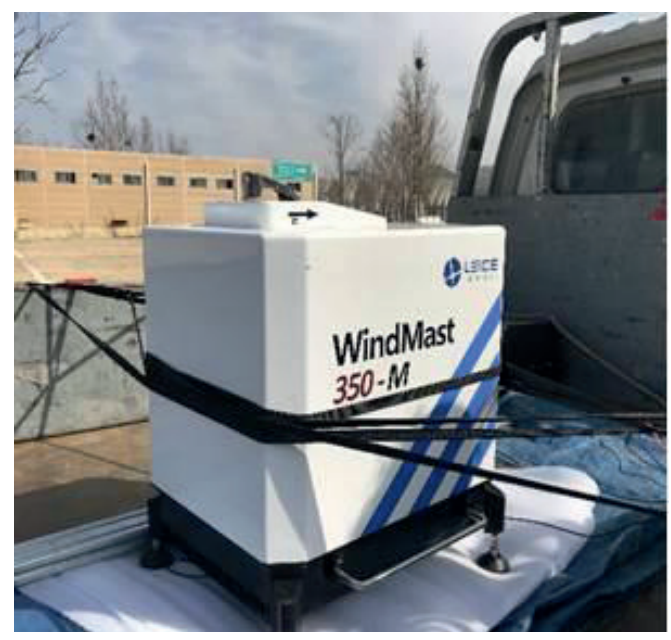

Figure 2. Floating Lidar System - WindMast 350-M
The total length of the circular route for vehicular motion experiment is about 570 meters. The height of zenith point (red star in Figure 1) is $75 \mathrm{~m}$ and the height of nadir point is $58 \mathrm{~m}$ (yellow star in Figure 1) in this route. The pickup truck was running at different speed between 15 to $30 \mathrm{~km} / \mathrm{h}$ and the attitude information (Yaw angle, Pitch angle and Roll angle). The motion velocity information (Eastward speed, Northward speed and Upward speed) and the height information of lidar system during the experiment could be acquired by GPS/MEMS with temporal resolution of $0.05 \mathrm{~s}$, which was used for motion correction and wind data retrieval.

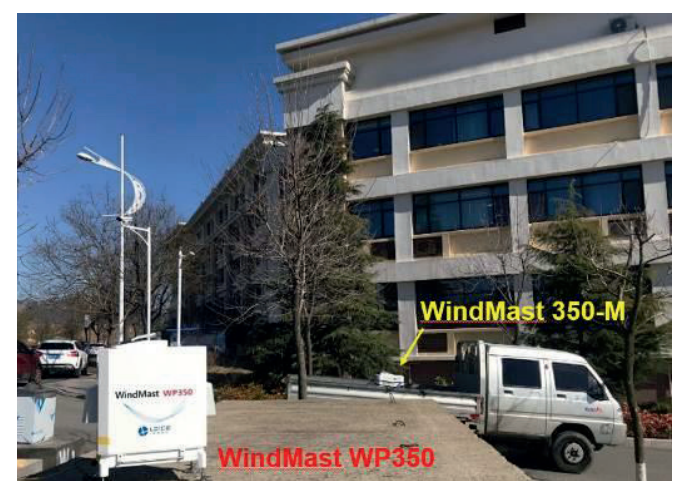

Figure 3. Comparison Experiment between WindMast 350-M and WindMast WP350 Systems

\subsection{Algorithm}

The recorded velocity corresponds to the relative velocity along the laser beam direction between the lidar and the atmospheric target. Considering the mobility of lidar system, the motion will add to the recorded radial velocity [2]. Therefore, a necessary step in data retrieval is the calibration of the radial velocity caused by the motion of lidar [3]. For this purpose, the real point angle (azimuth and pitch angle) of each laser radial need to be calculated first by combining the roll angle, pitch angle, yaw angle which are recorded by the GPS/MEMS and the recorded point angle of each laser radial [4].With the angle information, the projection of lidar motion velocity in each radial direction could be removed.

Afterwards, the real height of each range gate in all laser radial could be calculated with GPS height information. The radial velocity at selected altitude could be obtained by interpolation, which consists of the horizontal wind speed projection 
and the vertical speed projection in each radial direction, as shown in the equation:

$$
V_{\text {los }}=u \cdot \cos \alpha \cdot \cos \theta+v \cdot \sin \alpha \cdot \cos \theta+w \cdot \sin \theta
$$

$V_{l o s}$ is the LOS velocity in each direction, $\alpha$ is the azimuth angle and $\theta$ is the pitch angle of laser radial after correction. $u, v, w$ represent the north-south speed, east-west speed and vertical speed of wind. $u, v$ could be used to retrieve the wind horizontal speed and the wind direction.

\section{Results}

During the first vehicular motion experiment, different measuring range setting were used, more than 5 hours wind information was observed by WindMast 350-M and WindMast WP350. After the motion correction and the wind retrieval, the THI (Time-Height-Intensity) diagrams of the wind profiles measured by these two systems are shown as below.

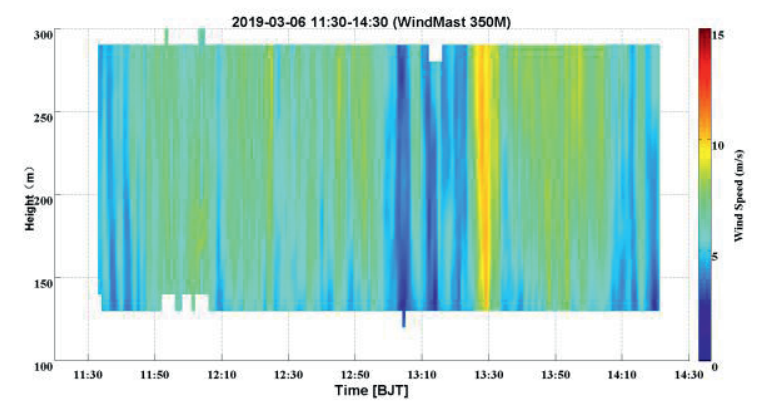

Figure 4. Wind THI diagram from 11:30 to 14:30BJT on 6 March 2019 observed by 350-M

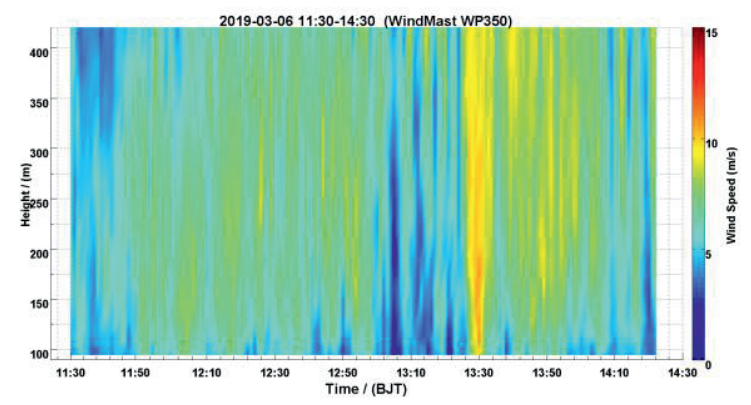

Figure 5. Wind THI diagram from 11:30 to 14:30BJT on 6 March 2019 observed by WP350

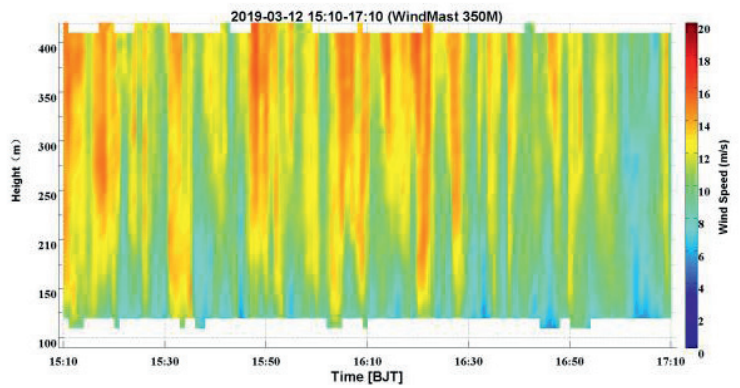

Figure 6. Wind THI diagram from 15:10 to 17:10BJT on 12 March 2019 observed by 350-M

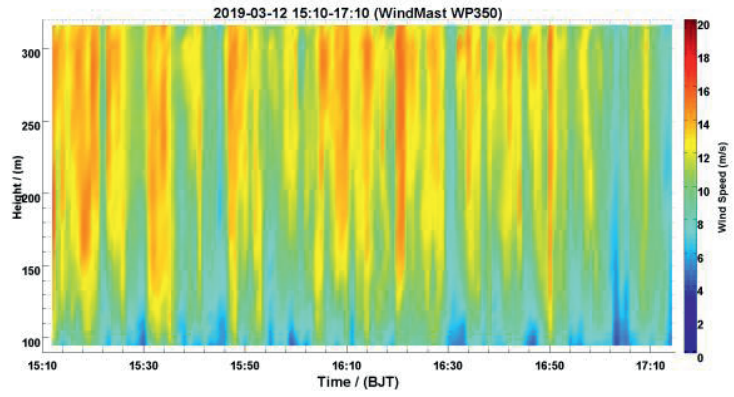

Figure 7. Wind THI diagram from 15:10 to 17:10BJT on 12 March 2019 observed by WP350

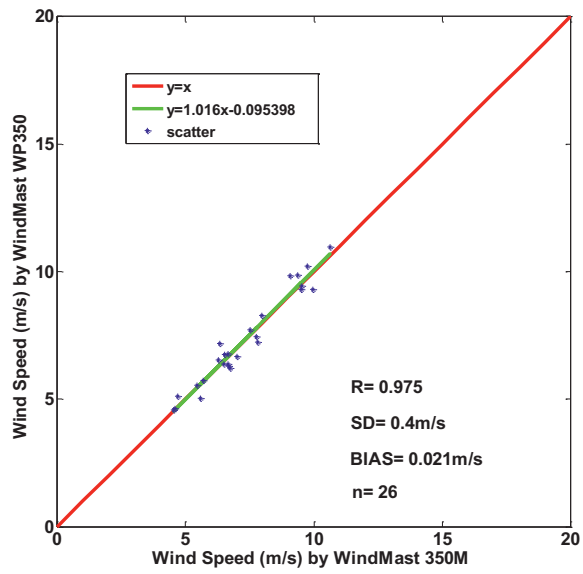

Figure 8. Scatter diagram of $150 \mathrm{~m}$ wind speed (10 mins) of 350-M and WP350

As presented in the THI diagrams, the wind profiles observed by $350-\mathrm{M}$ system fits well with the results from WP350 system after data correction and retrieval. 10-minute average horizontal wind speed at the height of 150 meters were selected for the statistical comparison in order to make more analysis of the wind speed details, as presented in Figure 8. The number of 
samples(n), standard deviation (SD), bias and correlation coefficient (R) are provided. The wind speed linear regression shows a correlation coefficient of 0.975 , SD of $0.4 \mathrm{~m} / \mathrm{s}$, demonstrating the reliability of WindMast 350-M system and the feasibility of the data correction and wind reversal method.

\section{Discussion and future works}

During the first vehicular motion experiment, carried by a pickup truck, the WindMast 350-M system was operating around the WindMast WP350 system. The longest distance between two systems is about 170 meters. There are some measurement deviations between these two systems, which were caused by the differences of location and laser pitch angle (WindMast 350-M: $60^{\circ}$, WindMast WP350: $71.38^{\circ}$ ). Due to the influence of the surrounding hill and buildings, the wind field of low-altitude area became inhomogeneous. Additionally, result from the limitation of the existing GPS/MEMS, the height error and the attitude error were induced into the wind retrieval.

Consequently, as an outlook, the further error analysis should be finalized in the following schedule.

\section{ACKNOWLEDGEMENTS}

We thank our colleagues for their great support during the field experiments, including Guangyao Dai, Yuanshuai Zhang from Ocean University of China and Xitao Wang, Caili Zhu, Tongtong Wang from QINGDAO Leice Transient Technology Co. LTD.

This research was supported by National Key Research and Development Program of China, grant number 2016YFC1400904 and the Fundamental Research Funds for the Central Universities, grant number 201822004.

\section{REFERENCES}

[1] Gottschall, J., et al. Energy Procedia 53: 156-161 (2014).

[2] X. Zhai, et al., Atmospheric Measurement Techniques, 11(3): 1313-1331 (2018)

[3] S. Wu, et al. EDP Sciences. EPJ Web of Conferences Vol. 176, p. 02012 (2017).
[4] Li Z. G., et al. Proceeding 'Dragon 3 Mid-Term Results Symposium', ESA SP-724, November (2014). 